\title{
Design of a Field Programmable Gate Array for Swarm Intelligent Controller Based on a Portable Robotic System: Review Study
}

Hanan A.R. Akkar, University of Technology, Iraq

Huthaifa Salman Khairy, University of Technology, Iraq

\begin{abstract}
Portable robots are considered an important device in many areas such as in medical, space research, emergency situations, applications, etc. The robots complete tasks efficiently and effectively without any human interaction. The most important advantages of portable robots are their small size and very high speed in problem processing with relatively high accuracy and efficiency compared with constant devices. In this paper, the authors discussed the applications of the robot systems based on swarm intelligent controller and field programmable gate array (FPGA). A component-oriented FPGA design platform is proposed for robot system integration because FPGAs are known to be power-efficient hardware platforms. From the results, they found that FPGA and swarm intelligence are very efficient in robotic systems and used in a wide area of applications.
\end{abstract}

\section{KEYWORDS}

Component-Oriented FPGA Design, FPGA, Portable Robot System, Swarm Intelligence

\section{INTRODUCTION}

Designing robust portable robot systems using traditional controllers is a complicated, time-wasting, resource-intensive process. However, providing the necessary flexibility and obtaining durability is represents a main challenges in the portable robotic control systems design models due to the mathematical complexity and it is necessary to consider any possible changes in the future. So that, we note the need to suggest effective devices to solve integrated optimization problems that meet real-time requirements. FPGAs are inexpensive and suitable techniques to exploit the basic parallelization of optimization algorithms to permit finding solutions in a very short time (Schmidt and Sass, 2010). These features make FPGAs convenient for robotic applications such as Portable robot applications, where optimization stages or adaptation processes must be implemented in a low energy consuming and short time.

Computing techniques which are recently evolutional have been used to solve complicated problems for embedded systems, in which the exhaustive search methods and the gradient-based are unpractical due to large execution time of the algorithms (Rao, 2009). Swarm intelligence like Particle Swarm Optimization (PSO) is an algorithm inspired from the social attitude of flocking birds and schooling fish paradigms (Kennedy, Poli, and Blackwell, 2007). For this reason, FPGAs can be used correctly to verify the parallelism found in the PSO algorithm not by realizing parallel particles only

\section{DOI: 10.4018/JCIT.20210401.oa6}

This article, published as an Open Access article on May 28th, 2021 in the gold Open Access journal, the Journal of Cases on Information Technology (converted to gold Open Access January 1st, 2021), is distributed under the terms of the Creative Commons Attribution License (http://creativecommons.org/licenses/by/4.0/) which permits unrestricted use, distribution, and production in any medium, provided the author of the original work and original publication source are properly credited. 
also could be by implementing as many as possible many concurrent calculations, giving performance amelioration in time execution. The additional advantage over PSO algorithm accuracy, it is speed toward to the optimum solution after a few iterations. However, for robotic systems applications particularly at the needing of hardware solutions, it is significant to stress the significance of utilizing simple processes for diversity, energy consumption and resource saving. Even using FPGA with a swarm intelligence will clearly improve the efficiency and performance of portable robotics systems.

\section{COMMON SYSTEM DESIGN}

Our proposed portable robotic system consists of the following parameters that shown in Figure 1. The system was consists of:

1. Field programmed gate array (FPGA)

2. Swarm intelligent controller (SIC)

3. Portable robot system

\subsection{Field Programmed Gate Array (FPGA)}

FPGA is an integrated circuit which could be programmed by a designer or a customer after industrialization. FPGA programming generally is defined by hardware description language (HDL). Circuit diagrams have previously been used to define configuration, but this is limited because of the emergence of electronic design automation tools. Methodology of design has faced several changes over the past decades. However, utilization of FPGA and VHDL for digital logic implementation has become prevalent in the past decade (Zhang, 2010).

Figure 1. proposed portable robotic system

\section{Start point}

\section{End point}

Port.

robot

SIC

FPGA
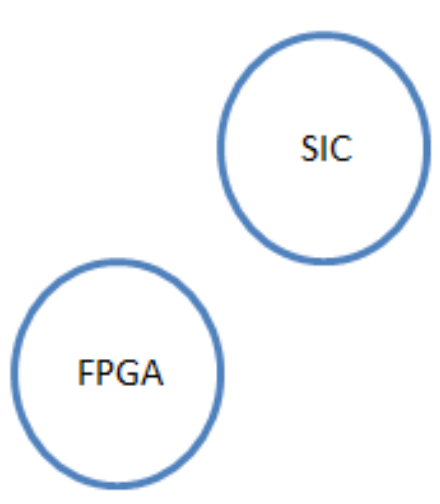
First FPGA presented at 1985 consists of 2,000 gates. The gate concentration increased to tens millions of gates from that period. During the increased concentration of FPGAs diversified device resources have turn into a standard merit of contemporaneous FPGA based devices. FPGA usage is still in the embedded systems in the evolution stage (Dubey, 2009). Many hardware resources provided by FPGAs Such as RAM, clock management modules, digital signal processing, embedded processors, and others that can achieve hardware solutions with low operating frequency.

\subsection{Swarm Intelligent Controller}

Swarm Intelligence (SI) is the artificial intelligence system, interested with multi factor intelligence systems design by inspiring the common behaviors of societies, insects and also the another animal societies. SI is considered a successful model the algorithm for complex problems and paid to very efficient clustering and optimization algorithms design. Most recent clustering algorithms which based on SI tools are Particle Swarm Optimization PSO and Ant Colony Optimization (ACO) (Chakraborty, 2008).

PSO is a new evolutionary computing technology introduced firstly by Eberhart and (Kennedy, 1995) It is a stochastic optimization approach, similar to the animals behavior such as a fish school, a birds, a flock of bees, or a group of people seeking a common goal in their lives. PSO also provides many required features such as ease of implementation, fewer mathematical requirements, parallel capabilities, and simplicity. PSO from the other hand has the advantages of being able to reach the optimum solution quickly so that it is adopted widely in the engineering applications.

\subsection{Portable Robot Systems}

Portable robots are becoming more popular in industrial and commercial settings. Hospitals use independent Portable robots to transport materials for many years ... etc. Portable robots represent a main focus of present research and every senior university may has one or more laboratories mostly focused on portable robot research (Ben-Tzvi, and Moubarak, 2011). In addition Portable robots found in military, security, and industrial settings. Indoor robots are include robots that able to do a certain domestic tasks like gardening or vacuuming as well as recreational robots.

Portable robot components are controller, sensors, actuators and controller software. The controller in general is a microprocessor, intelligent controller, embedded controller, and may be personal computer (PC). The sensors of portable robots depend on a robot requirements. The controller software may be either high-level languages like C, Pascal, VHDL, Fortran, assembly-level language, or special programs in real time. Also the requirements can be such as patient situation measurements, dead counts, proximity sensing, locating position, avoiding collision, and other applications (Tirunellayi and et. al. 2004).

\section{LITERATURE REVIEW}

The researchers goal is to develop methods that can realize the design of robot systems based on swarm intelligent and FPGA. They aim to improve the design capabilities which are used in various applications depending on the utilization place. The diversity of applications produces the improvement in the design methodologies such as the swarm intelligent algorithms. Various algorithms with FPGA were used that are shown in the following paragraphs:

Dipankar Chakrabarti, Shubhajit Roy Chowdhury and Hiranmay Saha develop a low-energy, low-cost, high-speed FPGA-based diagnostic system so as to detect in the doctor absence situation about the critical case in which the patient is approaching at an early stage is therefore appropriate for diagnosing patients at rural regions of developing countries that have rare doctors and rare energy availability. The diagnoses system can be constructed in health care centers in rural regions in which the patients able to register for diagnosis periodically and thus discover possible health risks early. Numerous different weights for pathophysiological parameters are contained in the diagnosis of a 
specific disease. To obtain these weights A new PSO called adaptive PSO has been suggested to find the optimal weights for pathophysiological parameters that gives more diagnosis accuracy. Intelligent systems based on FPGA has been implemented for detecting a patients' renal impairment early. BMI, urea, glucose, systolic, creatinine, renal diagnosis, and blood pressure diagnosis were viewed as a pathophysiological parameters. A critical approach of a patient detection has been validated by the tool using standard Cockford Gault equation for verifing the patient is really approaching a critical situation or not. The study on 80 patients population and achieving $97.5 \%$ accuracy of renal diagnosis by Bayesian analysis (Dipankar. Chowdhury, and Saha, 2008).

Chen-Chien Hsu, Shih-An Li,Chia-Jun Yu, and Ching-Chang Wong provides a hardware-software design using system on a programmable chip (SOPC) technique with the methods of pipeline design to enhance flexibility and performance of execution for PSO used in embedded applications. From the design architecture, the module of Particle Updating Accelerator by hardware realization for updating particles position and velocity and the module of Fitness evaluation realized on FPGA or a soft cored processor for objective functions evaluation are designed respectively to work together and closely to proceed the development process at several stages of design the suggested approach able to process several embedded applications optimization problems without necessity for hardware redesign because of the design flexibility. the PSO execution performance can be improved by designing a random number generator (RNG) with re-initialization scheme of particle to enhance exploration seek through the optimization process (Chien, Yu, and Chang, 2011).

Hsu-Chih Huang presents a parallel PSO algorithm known as PPSO with its application based on FPGA for structured environment autonomous robot for global path planning navigating with obstacles. The PPSO contains three PSOs connected in parallel with a communication operator in one FPGA chip. Advantages of parallel computing architecture are to obtaining better diversity of population and preventing early convergence compared with classical PSOs. Experimental results shown the Feature of the PPSO-FPGA path planner and fine global path planning navigation of autonomous robot (Hsu-Chih 2013).

Carlos H. Llanos, Daniel M. Muñoz, Mauricio Ayala-Rincón and Leandro dos S. Coelho suggested a new hardware architecture of new PSO algorithm applied to an artificial neural network (ANN) online training process by synthetic neural network robot controller to explore the parallel abilities of PSO algorithm. The hardware architecture invented relies on parallel opposition based PSO algorithm which is known as (HPOPSO). For simplifying hardware implementation Minor adjustments were declared in the premier OPSO. The architecture shown in Figure 2 was realized by a FPGA of Virtex 5 and operates by an particular floating-point arithmetic to allow a large dynamic range and high precision operations computation. The main goal of this job is to develop an embedded FPGA application suitable for OPSO, which is concentrated on the exploration efficiently of the essential parallel of OPSO algorithm via FPGAs. The diversity and growth of embedded system applications in multiple areas such as robotics, automation, medicine, and control among proposes to others making achieved objective of this work great interest (Llanos, and et. al. 2014).

Saikiran Ambati, Chinnaaiah.M., Sravanthi J, Manisha Yaddla, and Sanjay D have done research able to manage a robust internal environment with an effective hardware planner. This is done by using behavioral control in the multiplayer robot to implement navigation successfuliy. The goal of this robots is to access the target point that developed with the IR beacon, also to avoid indoor environments fixed and dynamic obstacles by presenting accurate algorithm for a different convex polygon type.Custom model robots are implemented by FPGA spartan 6, ultrasound sensor, RF2 transceiver, and infrared dialer. Where the suggested algorithm is realized through the use of efficient devices and found that the robots are moving to the goal by avoiding obstacles during the communication implicitly as shown in Figure 3 and this design is practical in the medical and industrial fields (Chinnaaiah, et. al. 2015).

Sendren Sheng-Dong Xu, Hsu-Chih Huang, and Chang Han Wu presents a SI for artificial immune system hybrid with Taguchi-genetic algorithm and its realized on FPGA to optimal inverse kinematics robotic operator using PC technology system. The hybridization strategy efficients the 
Figure 2. The general architecture of HPOPSO

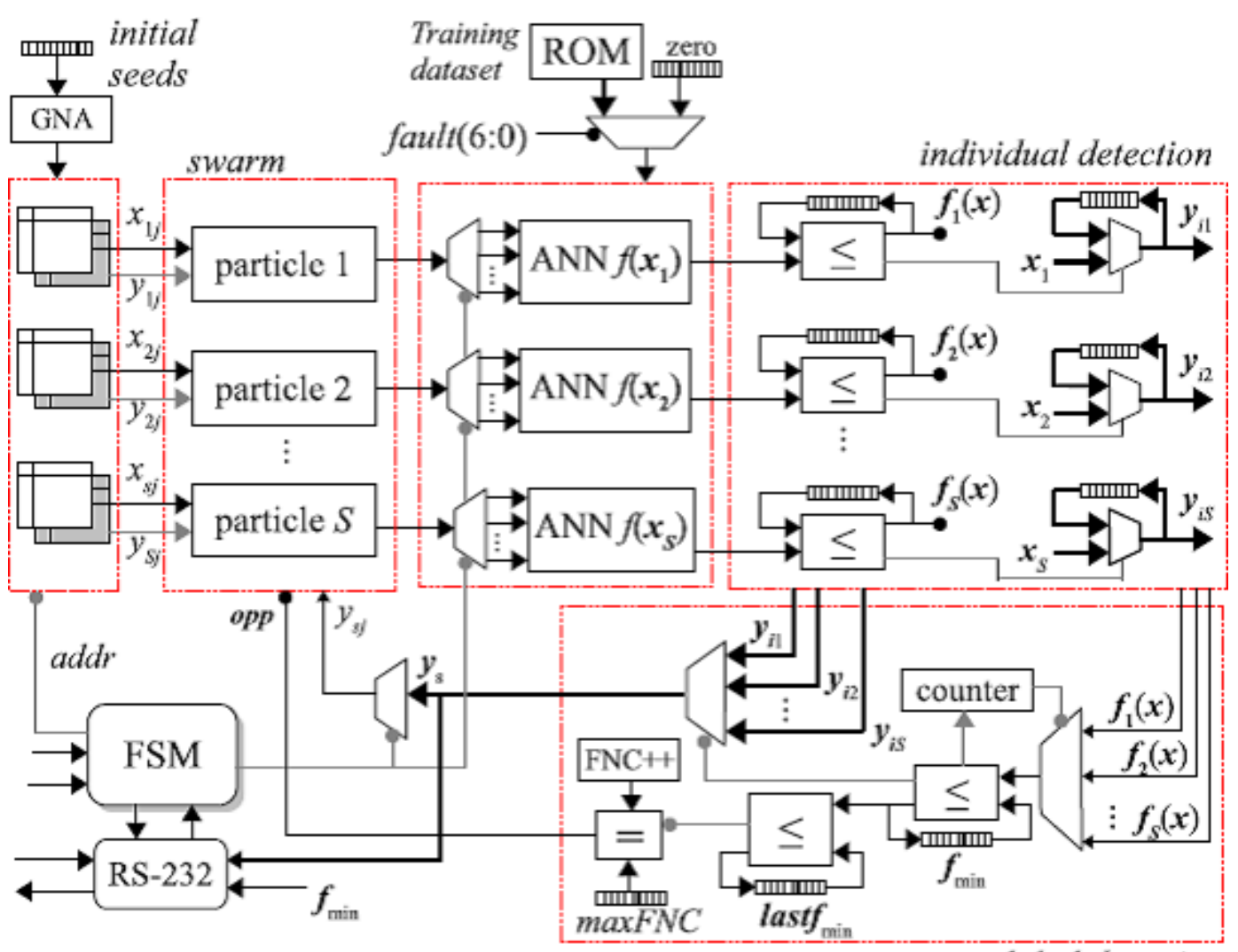

global detection

traditional artificial immune system model performance because of a Taguchi-genetic algorithm tuner. The realization based-FPGA of the suggested artificial immune system tuned with GA is more functional for embedded applications in practice. This system is then utilized to the 5-degree-offreedom robotic operator optimal inverse kinematics redundancy solver. The optimal joint shown in figure (4) is maintained when minimizing the predefined affinity function in the system tuned with GA for real world applications of embedded robotics (Sheng-Dong, and et. al. 2016).

B. Choudhury and Sandipan Pine aim to provide control strategy with the modified particle swarm optimization algorithm MPSO for a mobile robot. They were used FPGA for a real-time implementation to tracking a moving car based on the algorithm mentioned above. The simulation is done in Xilinx and MATLAB environment. The delays, power, the logic utilization and frequency is measured. After that the result is compared with existent PSO. The results showed the preferable of MPSO with FPGA than PSO-FPGA strategy in LUTs number and also for clock frequency and time (Pine, and Choudhury, 2020).

\section{DISCUSSION}

In this section we will show the most important results related with literature review of this work and discuss using FPGA and swarm intelligent activity in robot systems in a wide range diversity applications. There are many applications that uses swarm intelligent with field programmable gate array FPGA in its robotic system design as shown in Table 1. 


\section{Figure 3. Illustration of robot avoiding obstacles}

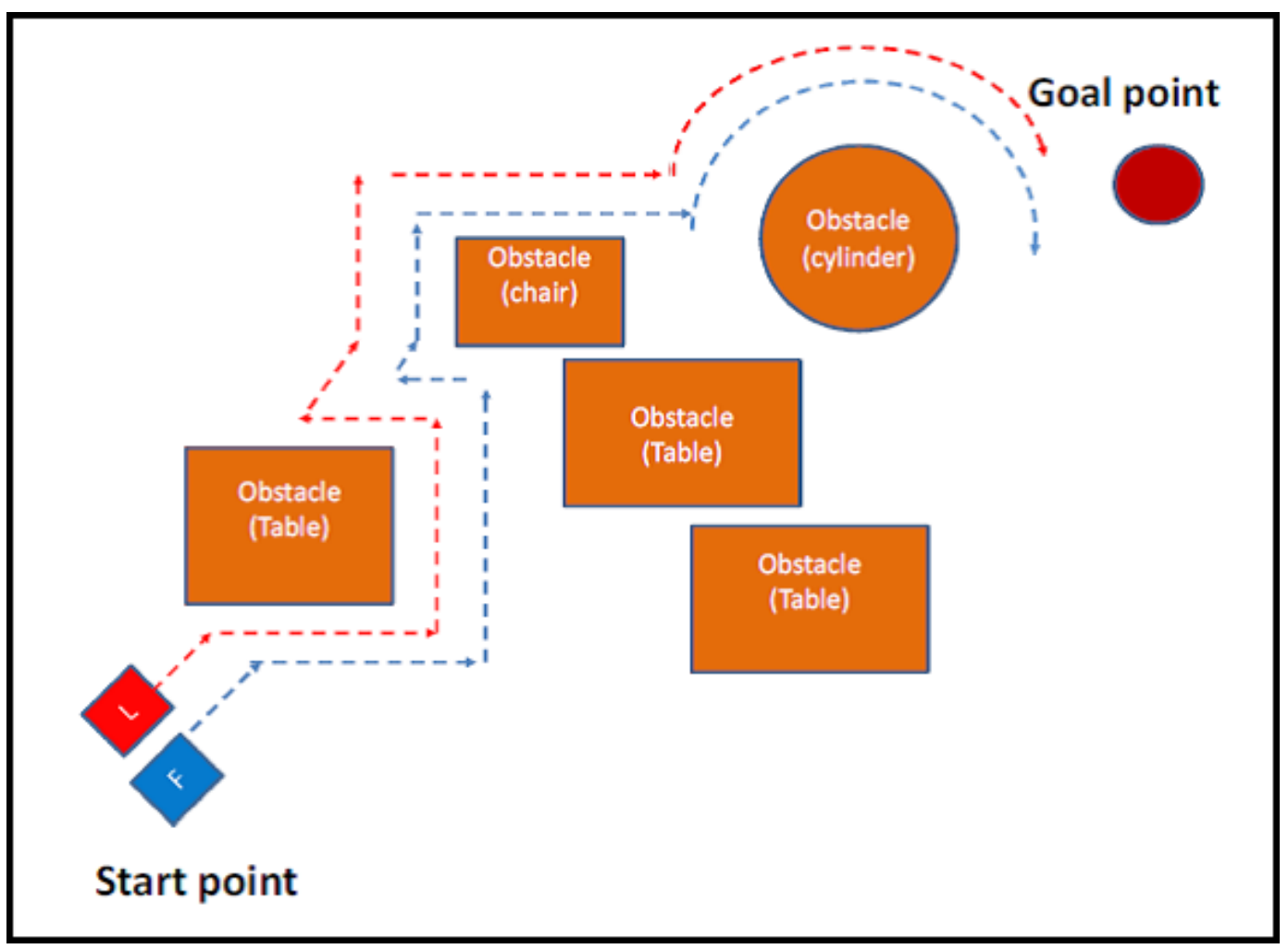

Figure 4. Joint trajectories of the 5-DOF robotic manipulator

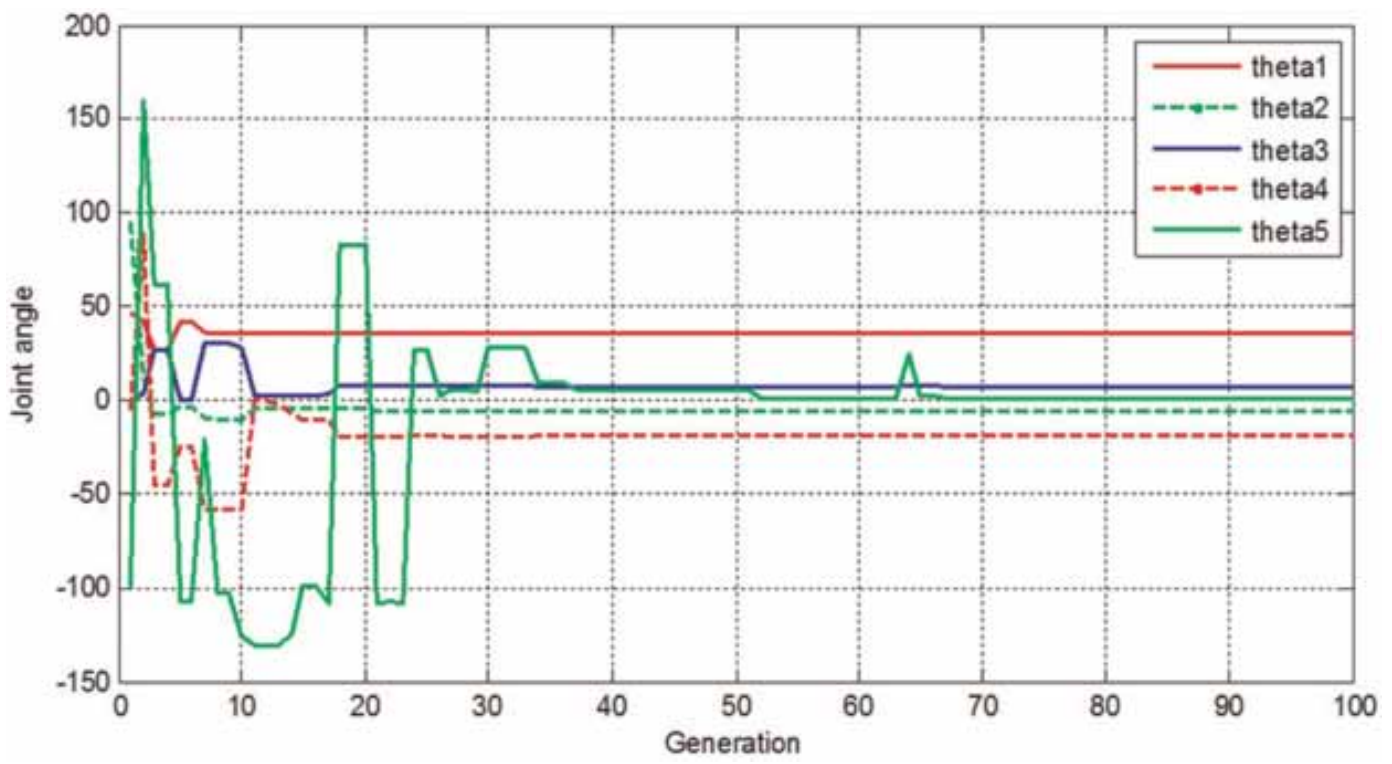


Table 1. Summary of robot systems design based on FPGA and SI

\begin{tabular}{|c|c|c|c|c|}
\hline Author Name & Year & Application type & Used technique & Accuracy \\
\hline $\begin{array}{l}\text { Hsu-Chih Huang1, Sendren } \\
\text { Sheng-Dong } \mathrm{Xu} \text {, and } \\
\text { Chang Han } \mathrm{Wu}\end{array}$ & 2016 & $\begin{array}{l}\text { Articulated industrial } \\
\text { robotic manipulator }\end{array}$ & $\begin{array}{l}\text { swarm intelligence tuned with } \\
\text { Taguchi-genetic algorithm and } \\
\text { field-programmable gate array }\end{array}$ & $\begin{array}{l}\text { The optimal joint } \\
\text { configuration is } \\
\text { obtained }\end{array}$ \\
\hline $\begin{array}{l}\text { Daniel M. Muñoz a, Carlos } \\
\text { H. Llanos, Leandro dos } \\
\text { S. Coelho, and Mauricio } \\
\text { Ayala-Rincón }\end{array}$ & 2014 & $\begin{array}{l}\text { novel hardware } \\
\text { architecture robot } \\
\text { controller }\end{array}$ & HPOPSO with FPGA & $\begin{array}{l}\text { Achieving } \\
\text { requirements of } \\
\text { embedded system } \\
\text { applications }\end{array}$ \\
\hline $\begin{array}{l}\text { Shih-An Li, Chen-Chien } \\
\text { Hsu, Ching-Chang Wong, } \\
\text { and Chia-Jun Yu }\end{array}$ & 2011 & $\begin{array}{l}\text { hardware/software } \\
\text { co-design approach for } \\
\text { embedded systems. }\end{array}$ & FPGA with PSO & $\begin{array}{l}\text { Efficient for } \\
\text { embedded } \\
\text { applications. }\end{array}$ \\
\hline $\begin{array}{l}\text { Sandipan Pine, and B. B. } \\
\text { Choudhury }\end{array}$ & 2020 & $\begin{array}{l}\text { Mobile robot control } \\
\text { strategy }\end{array}$ & MPSO with FPGA & $\begin{array}{l}\text { Good power, } \\
\text { frequency, delays, } \\
\text { and the logic } \\
\text { utilization }\end{array}$ \\
\hline $\begin{array}{l}\text { Shubhajit Roy Chowdhury, } \\
\text { Dipankar Chakrabarti \& } \\
\text { Hiranmay Saha }\end{array}$ & 2008 & Medical Diagnosis & APSO with FPGA & $\begin{array}{l}\text { study on } 80 \\
\text { patients with an } \\
\text { accuracy of up } \\
\text { to } 97.5 \% \text { in renal } \\
\text { diagnosis }\end{array}$ \\
\hline Hsu-Chih Huang & 2013 & $\begin{array}{l}\text { Global Path Planning } \\
\text { for Autonomous Robot } \\
\text { Navigation }\end{array}$ & PPSO with FPGA & $\begin{array}{l}\text { Efficient design } \\
\text { for global path } \\
\text { planning }\end{array}$ \\
\hline $\begin{array}{l}\text { Chinnaaiah.M.C, Saikiran } \\
\text { Ambati, Manisha Yaddla, } \\
\text { Sravanthi J, and Sanjay D }\end{array}$ & 2015 & $\begin{array}{l}\text { robust indoor } \\
\text { environment }\end{array}$ & PSO with FPGA & $\begin{array}{l}\text { Efficient in } \\
\text { industrial and } \\
\text { medical fields. }\end{array}$ \\
\hline
\end{tabular}

The medical diagnosis in (1) designed two automated diagnostic system to detect the patient condition early by using APSO and Fuzzy logic. The pathophysiological parameters weights utilized by medical diagnosis submitted using APSO and fuzzy logic and also applied on FPGA. The diagnostic performance system found to be better in comparession with recent processors. The accuracy of the diagnostic system is better than others obtained by fuzzy techniques as shown in Table 2 .

Articulated industrial robotic manipulator HW-SW co-design method to fulfill PSO to balance the usage flexibility and computational efficiency of PSO for embedded applications (2).

In (3) which presented a master-slave PPSO algorithm applicated for global path planning autonomous robot navigation. The system has been implemented efficiently into VHDL technique and SOPC technique with FPGA. From the results the FPGA-based PPSO system has an advantage compared with the conventional methods as shown in Figure 5.

Table 2. Accuracy comparative study of medical diagnosis

\begin{tabular}{|l|l|l|l|}
\hline \multicolumn{1}{|c|}{ Method } & \multicolumn{1}{|c|}{ Sensitivity } & \multicolumn{1}{c|}{ Specificity } & \multicolumn{1}{c|}{ Accuracy (\%) } \\
\hline APPSO & 1.0000 & 0.9629 & 97.5 \\
\hline PSO & 0.8461 & 0.9629 & 92.5 \\
\hline GA & 0.7692 & 0.9629 & 90 \\
\hline
\end{tabular}


Figure 5. Fitness values of GA, conventional PSO, and the FPGA-PPSO

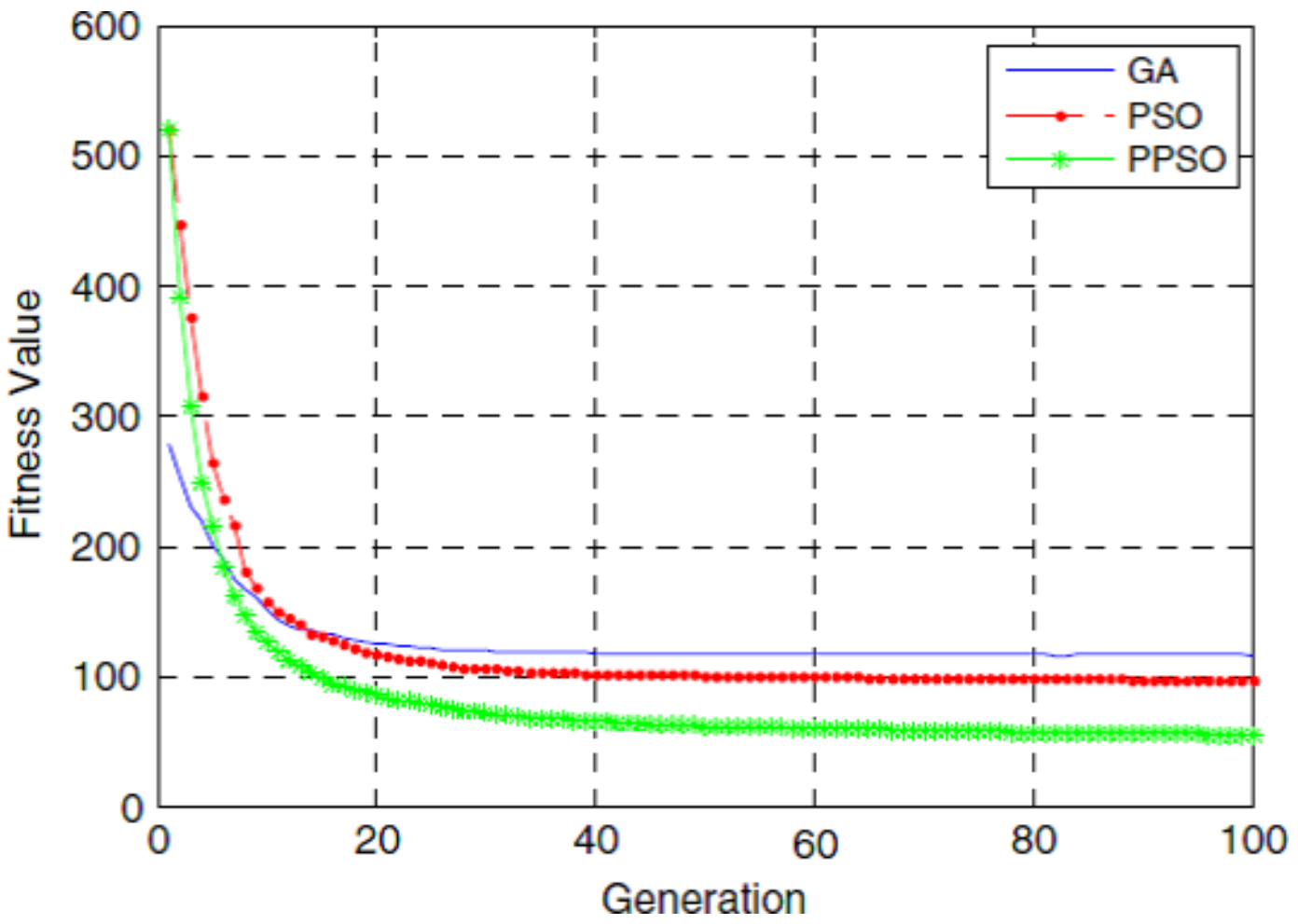

A novel hardware architecture robot controller in (4) of HPOPSO with FPGA is more efficient to Achieve the requirements of embedded system applications in compression with conventional PSO-FPGA.

Robust indoor environment system in (5) where the behaviour control of two robots and discusses the way to communicate with each other in doing the meticulous task synchronization in real time indoor environment to reach the goal point and it could be efficient in industrial and medical fields.

Articulated industrial robotic manipulator in (6) presented swarm intelligence tuned with Taguchigenetic algorithm and FPGA compared with PSO. The optimal joint configuration is obtained for AISTGA tuned with PSO.

In (7) there are two Mobile robot control strategies which are MPSO-FPGA and PSO-FPGA. Despite of the efficiency of strategies but that uses MPSO with FPGA is better than PSO-FPGA control strategy where MPSO used 417 total number of 4 input LUTs while PSO used 440 of 4 input LUTs also for clock frequency and time as shown in Table 3.

\section{CONCLUSION}

Swarm intelligent is distinguished from other methods used in artificial intelligence because its fast convergence to the solution as well as high efficiency and accuracy in finding the optimal solution to many problems related to robot systems. FPGA from the other side represents the best solution in robot systems design from the rest of the controllers due to its ease, simplicity and low cost in addition to its high speed and support for many sources such as digital signal processors and so on. However the use of swarm intelligent with FPGA in designing portable robot systems that are characterized 
Table 3. Proposed system performance compared with existing system

\begin{tabular}{|l|l|l|}
\hline \multicolumn{1}{|c|}{ Performance } & \multicolumn{1}{c|}{ MPSO } & \multicolumn{1}{c|}{ PSO } \\
\hline Minimum input arrival time & $3.990 \mathrm{~ns}$ & $3.498 \mathrm{~ns}$ \\
\hline Maximum output time & $4.694 \mathrm{~ns}$ & $4.700 \mathrm{~ns}$ \\
\hline Total number of 4 input LUTs & 417 & 440 \\
\hline
\end{tabular}

by their high speed and their ability to accomplish multiple functions in addition to that they have proven their worth when used in medical, engineering, industrial and other fields. 


\section{REFERENCES}

Abdulsahib, G. M., \& Khalaf, O. I. (2018). Comparison and Evaluation of Cloud Processing Models in CloudBased Networks. International Journal of Simulation-Systems, Science \& Technology, 19(5).

Alhayani \& Rane. (2014). Face recognition system by image processing. International Journal of Electronics and Communication Engineering \& Technology, 5(5), 80-90.

Alhayani, B., \& Ilhan, H. (2010). Efficient cooperative imge transmission in one-Way mult-hop sensor network. International Journal of Electrical Engineering Education, 6, 1-17.

Alhayani, B., \& Ilhan, H. (2017). Hyper spectral image classification using dimensionality reduction techniques. International Journal of Innovative Research in Electrical, Electronics, Instrumentation and Control Engineering, 5(4), 71-74. doi:10.17148/IJIREEICE.2017.5414

Alhayani, B., \& Ilhan, H. (2020). Image transmission over decode and forward based cooperative wireless multimedia sensor networks for Rayleigh fading channels in medical internet of things (MIoT) for remote health-care and health communication monitoring. Journal of Medical Imaging and Health Informatics, 10(1), 160-168. doi:10.1166/jmihi.2020.2691

Ben-Tzvi, P., \& Moubarak, P. M. (2011). Adaptive Manipulation of a Hybrid Mechanism Mobile Robot. IEEE. Chakraborty. (2008). Genetic and Evolutionary Computing. Information Sciences.

Chien, C., Li, S., \& Yu, C. (2011). Hardware/software co-design, for particle, swarm optimization algorithm. Information Sciences, 181(20), 4582-4596. doi:10.1016/j.ins.2010.07.017

Chinnaaiah, M. C., Sravanthi, J., \& Ambati, S. (2015). FPGA Based Robots Hardware Efficient Scheme For Real Time Indoor Environment With Behavioural Control. In IEEE Sponsored Second International Conference, on Innovations in Information, Embedded and Communication Systems. IEEE.

Dipankar, C., Chowdhury, S. R., \& Saha, H. (2008). Medical Diagnosis, Using Adaptive Perceptive Particle Swarm Optimization and Its Hardware Realization using Field Programmable Gate Array. Springer Science.

Dubey, R. (2009). Introduction to Embedded System Design Using Field Programmable Gate Arrays. Springer.

Hsu-Chih, H. (2013). FPGA-Based Parallel Metaheuristic PSO Algorithm and Its Application to Global Path Planning for Autonomous Robot Navigation. Springer.

Kennedy, J., \& Eberhart, R. (1995). Particleswarmoptimization. International Conference on Neural Networks, 1940-1948.

Kennedy, J., Poli, R., \& Blackwell, T. (2007). Particle Swarm Optimization An Overview. Springer.

Khalaf, O. I., \& Abdulsahib, G. M. (2019). Frequency estimation by the method of minimum mean squared error and P-value distributed in the wireless sensor network. Journal of Information Science and Engineering, 35(5), 1099-1112.

Khalaf, O. I., Abdulsahib, G. M., Kasmaei, H. D., \& Ogudo, K. A. (2020). A new algorithm on application of blockchain technology in live stream video transmissions and telecommunications. International Journal of e-Collaboration, 16(1), 16-32. doi:10.4018/IJeC.2020010102

Khalaf, O. I., Abdulsahib, G. M., \& Sadik, M. (2018). A Modified Algorithm for Improving Lifetime WSN. Journal of Engineering and Applied Sciences (Asian Research Publishing Network), 13, 9277-9282.

Khalaf, O. I., \& Sabbar, B. M. (2019). An overview on wireless sensor networks and finding optimal location of nodes. Periodicals of Engineering and Natural Sciences, 7(3), 1096-1101. doi:10.21533/pen.v7i3.645

Llanos, C. H., Munoz, D., Ayala-Rincon, M., \& Coelho, L. S. (2014). Hardware Opposition-based PSO Applied To Mobile Robot Controllers. Engineering Applications of Artificial Intelligence.

Pine, S., \& Choudhury, B. B. (2020). FPGA Implementation of Modified Swarm Optimization, Based Control Strategy for a Mobile Robot. Springer Nature Switzerland AG. doi:10.1007/978-3-030-30271-9_26 
Rao, S. (2009). Engineering Optimization: Theory and Practice (4th ed.). John Wiley and Sons. doi:10.1002/9780470549124

Schmidt, A. G., \& Sass, R. (2010). Embedded Systems Design with Platform FPGAs Principles and Practices. Elsevier Inc.

Sheng-Dong, S., Hsu-Chih, H., \& Chang, H. W. (2016). A Hybrid Swarm Intelligence of Artificial Immune System Tuned With Taguchi-Genetic Algorithm and its Field Programmable Gate Array Realization to Optimal Inverse Kinematics For an Articulated Industrial Robotic Manipulator. Advances in Mechanical Engineering, 8(1), 1-10.

Tirunellayi, S., Todkar, R., \& Gopalakrishnan, B. (2004). Design and Development of Anautonomous Mobile Smart Vehicle: A Mechatronics Application. Elsevier.

Zhang, P. (2010). Programmable-logic and application-specific integrated circuits (PLASIC). Advanced Industrial Control Technology. doi:10.1016/B978-1-4377-7807-6.10006-3

Hanan A. R. Akkar was born in Baghdad, Iraq. She received her B.Sc., M.Sc., and Ph.D. degrees in 1988, 1993, and 1998, respectively at Department of Electrical Engineering, University of Technology, Baghdad, Iraq. She is a Professor in Electronic Engineering at Department of Electrical Engineering, University of Technology, Baghdad, Iraq. She has been scientific publishing since 1992. She published more than 100 publications in national and international conferences and journals in fields of Switched-Capacitor Networks, Artificial Neural Network, Genetics Algorithm, Fuzzy Logic, Swarm Intelligent, FPGA, Robotics and Nano-Electronic.

Huthaifa Salman received the B.Sc. degree in Electrical Engineering from Tikrit University, Tikrit, Iraq, and the M.Sc. degree in electronics and communication engineering from Mosul University, Mosul, Iraq, from 2013 to 2017 he was joined in Ph.D. Research in 2018 in electronics and communication Department in University of Technology, Baghdad, Iraq. His general research interests lie in Artificial intelligent, Electronics, Swarm intelligence, PSO, communication theory and wireless communication networks and image processing, specific research area include compensation between them. 